\title{
Renal complications and subsequent mortality in acute critically ill patients without pre-existing renal disease
}

\author{
Shih-Ting Huang MD, Tai-Yuan Ke MD, Ya-Wen Chuang MD, Cheng-Li Lin MSc, Chia-Hung Kao MD
}

Cite as: CMAJ 2018 September 10;190:E1070-80. doi: 10.1503/cmaj.171382

\begin{abstract}
BACKGROUND: Most studies of longterm renal outcomes after acute critical illness have enrolled patients with preexisting renal dysfunction. We assessed renal outcomes in patients who did not have pre-existing renal disease and who were admitted to hospital for acute critical illness.
\end{abstract}

METHODS: We identified adults who did not have pre-existing renal disease and who were admitted to hospital for acute critical illness between 2000 and 2011, from the Taiwan National Health Insurance Research Database. Each patient was matched 1:2 with controls without acute critical illness, according to age, sex and index date. A subset was further matched 1:1 with controls using propensity scores. Outcomes included acute kidney injury, chronic kidney disease and end-stage renal disease.

RESULTS: We evaluated 33613 patients with acute critical illness matched to 63148 controls, of whom 14218 were propensity matched to 14218 controls. Patients with acute critical illness had incidence rates per 10000 person-years of 9.45 for acute kidney injury, 78.3 for chronic kidney disease and 21.0 for endstage renal disease. In the propensitymatched cohort, patients with acute critical illness had significantly higher risks of acute kidney injury (adjusted hazard ratio [aHR] 2.92, 95\% confidence interval $[\mathrm{Cl}]$ 1.78-4.77), chronic kidney disease (aHR 1.81, 95\% Cl 1.57-2.08), and end-stage renal disease (aHR 3.60, $95 \% \mathrm{Cl} 2.50-5.18)$. Acute critical illness conferred higher mortality risk among patients who subsequently developed end-stage renal disease (aHR 3.37, $95 \% \mathrm{Cl} 2.07-5.49)$ or chronic kidney disease (aHR 2.16, 95\% Cl 1.67-2.80).

INTERPRETATION: Patients with acute critical illness and without pre-existing renal disease have a higher risk of adverse renal outcomes and subsequent mortality. A resolved episode of critical illness has implications for future renal function surveillance, even in patients without pre-existing renal disease.
A cute kidney injury ${ }^{1}$ is prevalent in patients with acute critical illness, with $53.2 \%$ in patients with severe sepsis, $12.9 \%$ in patients with acute myocardial infarction and $20.9 \%$ in patients with stroke. ${ }^{2-4}$ The incidence of acute kidney injury has risen substantially in the last decade, associated with the burden of acute critical illnesses, such as septicemia, respiratory failure and shock. ${ }^{5,6}$ The high prevalence of acute kidney injury in patients with acute critical illness is explained by acute physiologic abnormalities that include peripheral vasodilation, decreased cardiac output and intravascular fluid volume, which attenuate renal function reversibility even in patients with previously preserved renal function. ${ }^{7}$ Notably, about $30 \%$ of patients with acute critical illness have preadmission renal dysfunction. ${ }^{8}$ In the setting of acute critical illness, acute kidney injury requiring dialysis has been associated consistently with in-hospital mortality and morbidities, such as chronic kidney disease and end-stage renal disease. ${ }^{9-12}$

For long-term renal complications, chronic kidney disease is the most prevalent renal dysfunction. ${ }^{13,14}$ Patients with chronic kidney disease have a high rate of progression to end-stage renal disease, cardiovascular disease and mortality. ${ }^{14}$ Among patients admitted to hospital, the risk of end-stage renal disease is the highest in those with acute-on-chronic kidney disease, followed by those with acute kidney injury and those with chronic kidney disease, relative to patients without kidney diseases. ${ }^{15,16}$

Recently, acute kidney injury and chronic kidney disease have been recognized as related disease entities in that they represent a continuum of the disease process. The term "acute kidney disease" is used to define the disease course after acute kidney injury in patients with acute critical illness whose pathophysiologic 
processes are ongoing. ${ }^{17}$ Thus, nephrologists are recommended to keep patients who have acute critical illness and acute kidney injury or chronic kidney disease under surveillance for risk of subsequent end-stage renal disease. . $^{2,18}$

Despite robust evidence on acute and chronic renal risks in patients with acute critical illness, most studies have enrolled patients with pre-existing renal dysfunction. ${ }^{9-16}$ Information about whether patients with acute critical illness without preexisting kidney disease have adverse renal outcomes is scarce. We aimed to find out whether these patients carry long-term renal risks compared with patients with nonacute critical illness.

\section{Methods}

\section{Data source}

This retrospective cohort study used data from Taiwan's Longitudinal Health Insurance Database 2000 (LHID2000) of the National Health Insurance Research Database (NHIRD) from Jan. 1, 2000, to Dec. 31, 2011. The NHIRD, including all beneficiaries' inpatient and outpatient medical claims, enrolled data from 23 million Taiwanese people in 2014 (99.9\% of the population). ${ }^{19,20}$ The LHID2000 database comprises 1000000 beneficiaries randomly selected from the National Health Insurance 2000 Registry for Beneficiaries. ${ }^{21}$ Definitions of diseases in the LHID are based on the International Classification of Diseases, Ninth Revision, Clinical Modification (ICD-9-CM).

\section{Study design and participants}

We identified inpatients with the following newly diagnosed critical illnesses: septicemia (ICD-9-CM 038), septic shock (ICD-9-CM 785.52), acute myocardial infarction (ICD-9-CM 410), stroke (ICD9-CM 430-432) and shock (ICD-9-CM 785.59, 998.09, and 958.4) (Figure 1). We set the date of the diagnosis of critical illness as the index date. We excluded patients with previous inpatient and outpatient history of acute kidney injury (ICD-9-CM 584.5, 584.6, $584.7,584.8$, and 584.9), chronic kidney disease (stage G1-5; ICD9-CM 585.1-581.5) and end-stage renal disease (ICD-9-CM 586), and patients younger than 20 years. We also excluded patients with incident acute kidney injury, chronic kidney disease or endstage renal disease within 90 days of the index date. Finally, we enrolled patients with acute critical illness without pre-existing renal disease as the study cohort.

The control cohort was randomly selected from the database of inpatients and outpatients without acute critical illness, using the same exclusion criteria as the control cohort. Each patient with acute critical illness was frequency matched by age (a span of every 5 years), sex and index year with control patients, at a 1:2 ratio. We also performed propensity-score matching between

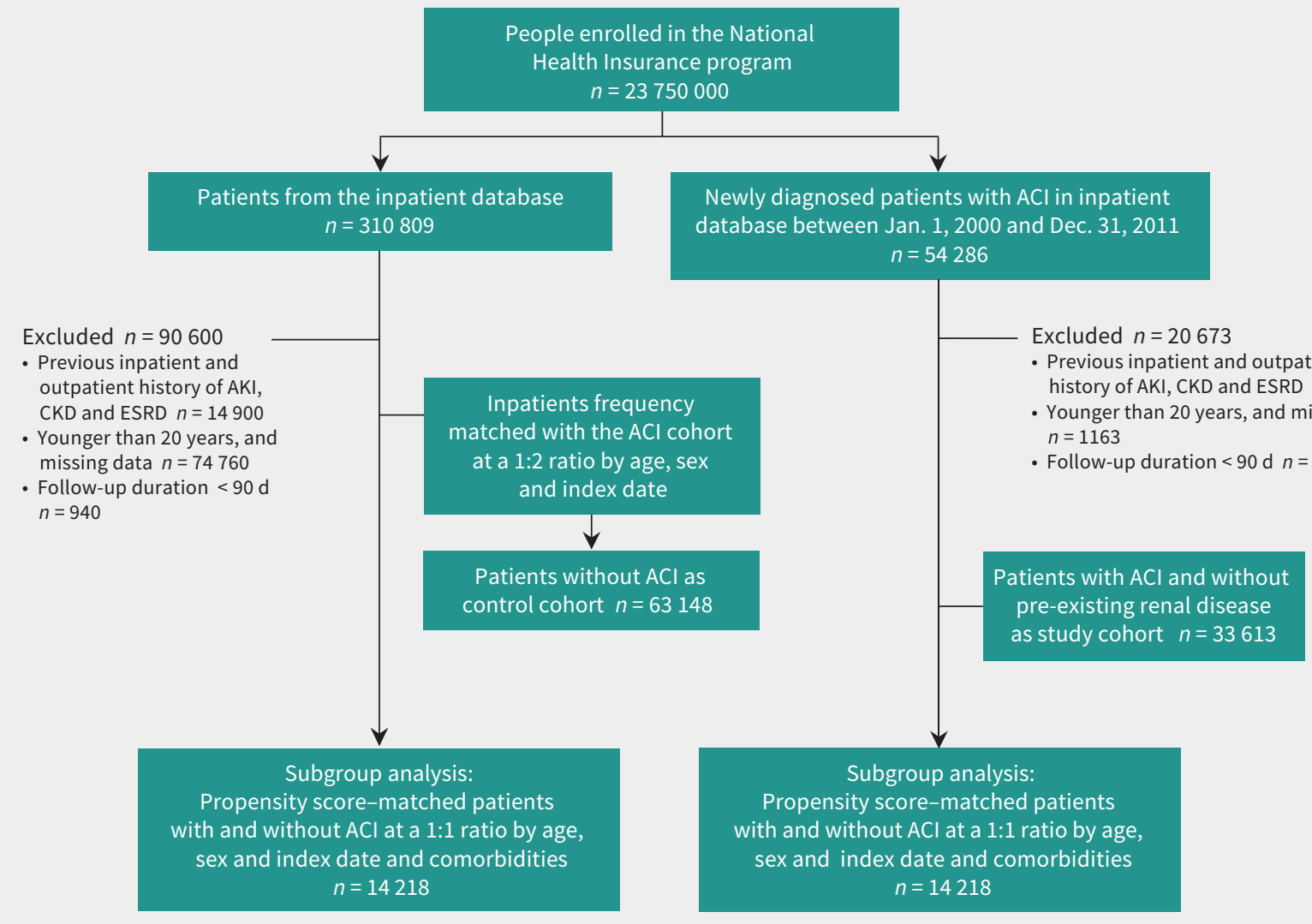

Figure 1: Flowchart of study design and patient selection. Note: $\mathrm{ACl}=$ acute critical illness, $A K I=$ acute kidney injury, $C R D=$ chronic renal disease, $E S R D=$ end-stage renal disease. 
the acute critical illness and nonacute critical illness cohorts by gender, age, index year and underlying comorbidities at a 1:1 ratio, to reduce the risk of confounding effect.

The follow-up period commenced from the index date until the occurrence of renal complications, the date on which the patients were censored because of withdrawal from the National Health Insurance program, or the end of the study (Dec. 31, 2011), whichever occurred first.

\section{Demographic and comorbid variables}

We obtained demographic information and pre-existing comorbidities that affect renal outcomes, such as diabetes, hypertension, cirrhosis, hyperlipidemia, coronary artery disease, congestive heart failure and cancer. We also identified potential confounders of critical illness and the composite renal outcome such as Charlson Comorbidity Index score, stay in the intensive care unit (ICU) or mechanical ventilation. $., 6,8,22,23$

Table 1: Baseline characteristics of patients admitted to hospital (2000-2011), by the presence of critical illness, and after matching for age, sex and index date, or for propensity score ${ }^{\star}$

Acute critical illness

\begin{tabular}{|c|c|c|c|c|c|c|}
\hline \multirow[b]{2}{*}{ Variable } & \multicolumn{2}{|c|}{ Age- and sex-matched } & \multirow[b]{2}{*}{$p$ value $\ddagger$} & \multicolumn{2}{|c|}{ Propensity score-matched } & \multirow{2}{*}{$\begin{array}{c}\text { Standardized } \\
\text { mean } \\
\text { differences§ }\end{array}$} \\
\hline & $\begin{array}{c}\text { No } \\
n=63148(\%) \dagger\end{array}$ & $\begin{array}{c}\text { Yes } \\
n=33613(\%) \dagger\end{array}$ & & $\begin{array}{c}\text { No } \\
n=14218(\%) \dagger\end{array}$ & $\begin{array}{c}\text { Yes } \\
n=14218(\%) \dagger\end{array}$ & \\
\hline Age, yr & & & 0.001 & & & \\
\hline$\leq 49$ & $12928(20.5)$ & $6464(19.2)$ & - & $2327(16.4)$ & $3104(21.8)$ & 0.14 \\
\hline $50-64$ & $18578(29.4)$ & $9289(27.6)$ & - & $3747(26.4)$ & $3486(24.5)$ & 0.04 \\
\hline $65+$ & $31642(50.1)$ & $17860(53.1)$ & - & $8144(57.3)$ & $7628(53.7)$ & 0.07 \\
\hline Mean \pm SD & $61.9 \pm 15.1$ & $64.1 \pm 15.8$ & $<0.001$ & $64.1 \pm 15.0$ & $63.6 \pm 17.1$ & 0.03 \\
\hline Sex & & & 0.28 & & & \\
\hline Female & $26485(41.9)$ & $14218(42.3)$ & - & $5972(42.0)$ & $5988(42.1)$ & 0.002 \\
\hline Male & $36663(58.1)$ & $19395(57.7)$ & - & $8246(58.0)$ & $8230(57.9)$ & 0.002 \\
\hline \multicolumn{7}{|l|}{ Critical illness } \\
\hline Septicemia, septic shock & - & $13468(40.1)$ & - & - & 7746 & - \\
\hline AMI & - & $2324(6.91)$ & - & - & 524 & - \\
\hline Stroke & - & $16921(50.3)$ & - & - & 5327 & - \\
\hline Other shock & - & $900(0.27)$ & - & - & 621 & - \\
\hline CCI score§ & & & $<0.001$ & & & \\
\hline 0 & $54664(86.6)$ & $6430(19.1)$ & - & $5906(41.5)$ & 6200 (43.6) & 0.04 \\
\hline 1 & $6671(10.6)$ & $17517(52.1)$ & - & $6499(45.7)$ & $6156(43.3)$ & 0.05 \\
\hline 2 or more & $1813(2.87)$ & $9666(28.8)$ & - & $1813(12.8)$ & $1862(13.1)$ & 0.01 \\
\hline \multicolumn{7}{|l|}{ Comorbidity } \\
\hline Diabetes & $6778(10.7)$ & 8031 (23.9) & $<0.001$ & 2403 (16.9) & $2346(16.5)$ & 0.01 \\
\hline Cirrhosis & $13176(20.9)$ & $7800(23.2)$ & $<0.001$ & $3647(25.7)$ & $3432(24.1)$ & 0.04 \\
\hline Hypertension & $26883(42.6)$ & $22579(67.2)$ & $<0.001$ & 7695 (54.1) & 7730 (54.4) & 0.01 \\
\hline Hyperlipidemia & $14924(23.6)$ & $10510(31.3)$ & $<0.001$ & $3878(27.3)$ & 3897 (27.4) & 0.003 \\
\hline CAD & $13300(21.1)$ & 12391 (36.9) & $<0.001$ & $4235(29.8)$ & 4203 (29.6) & 0.01 \\
\hline $\mathrm{CHF}$ & 2963 (4.69) & 3951 (11.8) & $<0.001$ & $1337(9.40)$ & $1312(9.23)$ & 0.01 \\
\hline COPD & $10154(16.1)$ & $7532(22.4)$ & $<0.001$ & $3553(25.0)$ & $3340(23.5)$ & 0.04 \\
\hline Cancer & $2790(4.42)$ & $2144(6.38)$ & $<0.001$ & $1076(7.57)$ & $1022(7.19)$ & 0.02 \\
\hline Admission to ICU & $2800(4.43)$ & 11969 (35.6) & $<0.001$ & 2470 (17.4) & 2746 (19.3) & 0.05 \\
\hline Mechanical ventilation & $1430(2.26)$ & $5666(16.9)$ & $<0.001$ & $1209(8.50)$ & $1387(9.76)$ & 0.04 \\
\hline
\end{tabular}

Note: $\mathrm{AMI}=$ acute myocardial infarction, $\mathrm{CAD}=$ coronary artery disease, $\mathrm{CCI}=$ Charlson Comorbidity Index, $\mathrm{CHF}=$ congestive heart failure, $\mathrm{COPD}=$ chronic obstructive pulmonary disease, $\mathrm{ICU}=$ intensive care unit, $\mathrm{SD}=$ standard deviation.

*Propensity scores were calculated based on sex, age, index year and underlying comorbidities.

tUnless otherwise specified.

‡All $p$ values are based on the $\mathrm{X}^{2}$ test except for mean age, which is based on the $t$ test.

$\S A$ standardized mean difference of $\leq 0.10$ indicates a negligible difference between the 2 cohorts. 


\section{Outcomes}

Primary outcomes were diagnosis of acute kidney injury, chronic kidney disease or end-stage renal disease in the follow-up period. We identified patients who fulfilled the criteria of acute kidney injury according to the clinical practice guideline from the Kidney Disease Improving Global Outcomes, ${ }^{1}$ using ICD-9-CM. Clinically recognized chronic kidney disease is defined according to the criteria in the clinical practice guideline from the Kidney Disease Outcomes Quality Initiative. ${ }^{24}$ Patients with end-stage renal disease were identified if they were registered in the catastrophic illness registry, indicating dialysis dependence for more than 3 months.
We evaluated the effect of acute critical illness status on mortality, modified by time-dependent acute kidney injury, chronic kidney disease or end-stage renal disease as the secondary end point. The accuracy of acute kidney injury, chronic kidney disease and end-stage renal disease diagnoses recorded in our claims data has been validated. ${ }^{25,26}$

\section{Statistical analysis}

We compared demographic characteristics and comorbidities in the study and control cohorts using 2 models: a frequencymatched and a propensity score-matched model. In the frequencymatched model, continuous data are reported as mean and

Table 2: Incidence and hazard ratio of acute kidney injury, chronic kidney disease and end-stage renal disease in patients with critical illness compared with those without, by type of matching

\section{Critical illness}

\begin{tabular}{|c|c|c|c|c|}
\hline \multirow[b]{2}{*}{ Outcome } & \multicolumn{2}{|c|}{ Age- and sex-matched } & \multicolumn{2}{|c|}{ Propensity score-matched } \\
\hline & $\begin{aligned} & \text { No } \\
n= & 63148\end{aligned}$ & $\begin{array}{c}\text { Yes } \\
n=33613\end{array}$ & $\begin{aligned} & \text { No } \\
n= & 14218\end{aligned}$ & \begin{aligned} \multicolumn{1}{c}{ Yes } \\
$n=14218\end{aligned}$ \\
\hline \multicolumn{5}{|l|}{ Acute kidney injury } \\
\hline Follow-up time (year, mean \pm SD) & $5.94 \pm 3.17$ & $4.75 \pm 3.20$ & $5.40 \pm 3.08$ & $4.76 \pm 3.21$ \\
\hline No. of events & 88 & 151 & 23 & 53 \\
\hline Person-years & 375239 & 159836 & 76747 & 67635 \\
\hline Rate $^{\star}$ & 2.35 & 9.45 & 3.00 & 7.84 \\
\hline Crude HR (95\% CI) & 1 (Reference) & $4.16(3.20-5.42)$ & 1 (Reference) & $2.65(1.62-4.32)$ \\
\hline Adjusted HR† (95\% CI) & 1 (Reference) & $2.84(1.95-4.15)$ & 1 (Reference) & $2.92(1.78-4.77)$ \\
\hline \multicolumn{5}{|l|}{ Chronic kidney disease } \\
\hline Follow-up time (year, mean \pm SD) & $5.88 \pm 3.16$ & $4.66 \pm 3.17$ & $5.33 \pm 3.07$ & $4.67 \pm 3.19$ \\
\hline No. of events & 1402 & 1225 & 325 & 484 \\
\hline Person-years & 371108 & 156556 & 75818 & 66345 \\
\hline Rate $^{\star}$ & 37.8 & 78.3 & 42.9 & 73.0 \\
\hline Crude HR $(95 \% \mathrm{Cl})$ & 1 (Reference) & $2.13(1.97-2.30)$ & 1 (Reference) & $1.72(1.50-1.98)$ \\
\hline Adjusted HR† (95\% Cl) & 1 (Reference) & $1.81(1.61-2.04)$ & 1 (Reference) & $1.81(1.57-2.08)$ \\
\hline \multicolumn{5}{|l|}{ End-stage renal disease } \\
\hline Follow-up time (year, mean \pm SD) & $5.94 \pm 3.17$ & $4.75 \pm 3.19$ & $5.40 \pm 3.08$ & $4.75 \pm 3.21$ \\
\hline No. of events & 184 & 335 & 39 & 116 \\
\hline Person-years & 375010 & 159607 & 76717 & 67570 \\
\hline Rate $^{\star}$ & 4.91 & 21.0 & 5.08 & 17.2 \\
\hline Crude HR $(95 \% \mathrm{CI})$ & 1 (Reference) & $4.65(3.88-5.56)$ & 1 (Reference) & $3.49(2.43-5.01)$ \\
\hline Adjusted HR† (95\% CI) & 1 (Reference) & $3.26(2.52-4.22)$ & 1 (Reference) & $3.60(2.50-5.18)$ \\
\hline \multicolumn{5}{|c|}{ 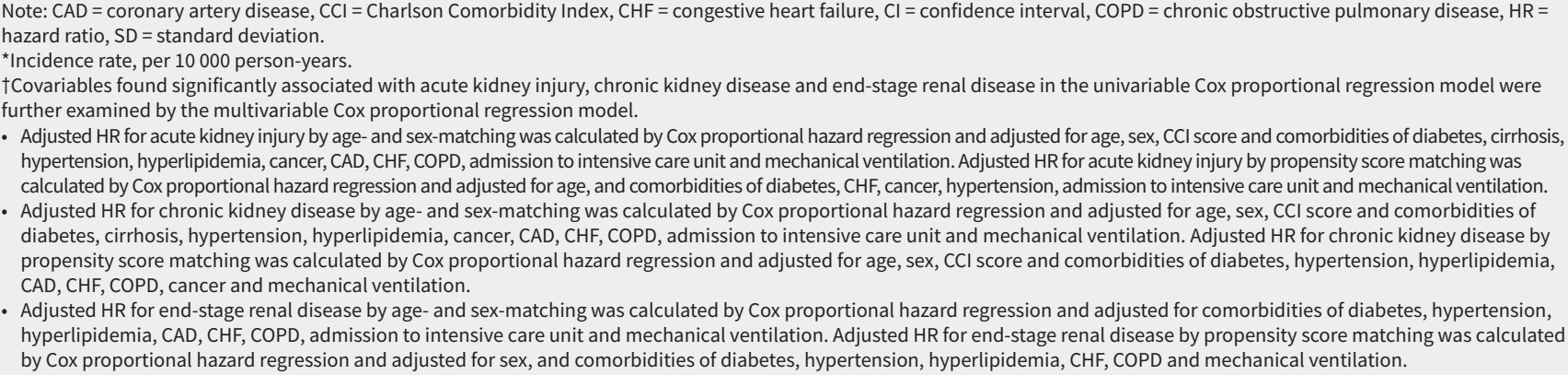 } \\
\hline
\end{tabular}


standard deviation (SD). Categorical data are expressed as counts and percentages. $X^{2}$ and Student $t$ tests were used for comparing dichotomous and continuous variables, respectively, between the 2 cohorts. A 2-sided $p$ value of less than 0.05 was considered significant. In the propensity score-matched model, we used logistic regression to estimate the risk of diseases by calculating the propensity score in both cohorts in the propensity score-matching process. ${ }^{27}$ Baseline characteristics of the acute critical illness and control cohorts were compared using standardized mean differences. Values of standardized mean differences $\leq 0.10$ showed a negligible difference in mean values between the 2 cohorts.

We calculated the cumulative incidence of the first diagnosis of primary renal outcome using the Kaplan-Meier method, and used the log-rank test to verify the equality of survivor functions between study groups. We used Cox proportional hazards regression models to determine the effects of acute critical illness on the risks of acute kidney injury, chronic kidney disease or endstage renal disease and the effects of acute critical illness status on mortality in patients with acute critical illness and nonacute critical illness in the frequency- and propensity score-matched cohorts. Because death is a competing risk for renal outcomes as a function of critical illness, we analyzed a competing risk model to estimate the subhazard ratios (SHRs) and 95\% confidence intervals (Cls) of the renal outcomes. ${ }^{28}$ Covariables significantly associated with outcome in the univariable competing-risks regression model were further examined by multivariable regression model. We used SAS (Version 9.3; SAS Institute, Inc., Cary, NC) for all statistical analyses.

\section{Ethics approval}

Patient records were anonymized, and this study was approved for exemption from informed consent rules by the Ethics Review Board of China Medical University and Hospital in Taiwan (CMUH104-REC2-115-CR2).

\section{Results}

Our study included 33613 patients with acute critical illness without pre-existing renal disease, matched with 63148 controls. Of these, 14218 patients with acute critical illness were matched with 14218 controls using propensity scores. The frequency-matched acute critical illness cohort predominantly included patients who were older than 65 years (53.1\%), were men (57.7\%) and had hypertension (67.2\%) and coronary artery disease (36.9\%). Sepsis and stroke were the leading causes of critical illnesses in the acute critical illness cohort, with a high prevalence of ICU stay (35.6\%) and mechanical ventilation (16.9\%). In the propensity score-matched model, the acute critical illness and nonacute critical illness cohorts had similar baseline characteristics (Table 1).

\section{Primary outcome}

The mean follow-up period for patients developing acute kidney injury, chronic kidney disease and end-stage renal disease in the frequency-matched acute critical illness cohort was mean \pm SD $4.75 \pm 3.20,4.66 \pm 3.17$, and $4.75 \pm 3.19$ years, respectively (Table 2). Acute kidney injury was more frequent in the acute
A
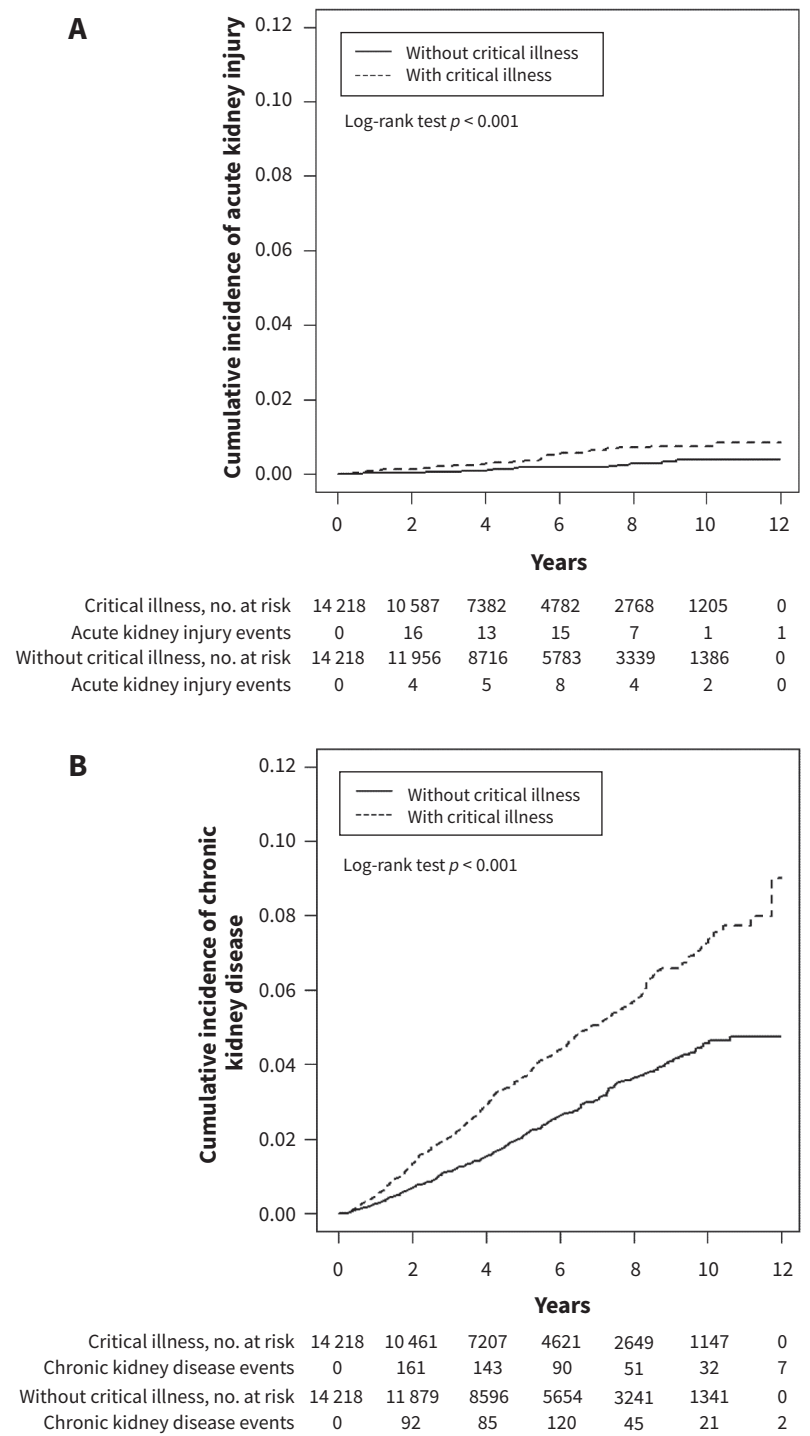

\section{C}

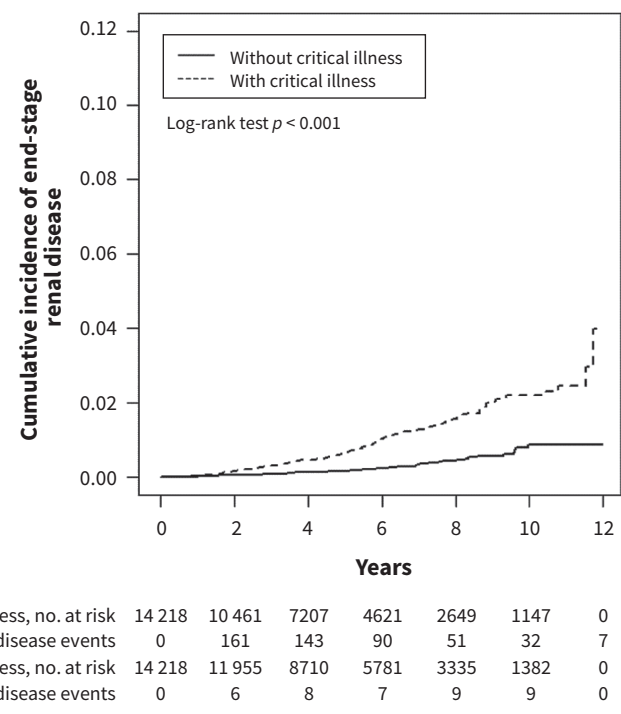

Figure 2: Cumulative incidence of acute kidney injury (A), chronic kidney disease (B) and end-stage renal disease (C) in individuals with and without critical illness by propensity-score matching. 
Table 3: Incidence and subhazard ratio of acute kidney injury, chronic kidney disease and end-stage renal disease in propensity score-matched cohorts, using competing-risks regression models

\begin{tabular}{|c|c|c|}
\hline \multirow[b]{3}{*}{ Outcome } & \multicolumn{2}{|c|}{ Competing-risks regression models } \\
\hline & \multicolumn{2}{|c|}{ Critical illness } \\
\hline & $\begin{array}{c}\text { No } \\
n=14218(95 \% \mathrm{Cl})\end{array}$ & $\begin{array}{c}\text { Yes } \\
n=14218(95 \% \mathrm{Cl})\end{array}$ \\
\hline \multicolumn{3}{|c|}{ Acute kidney injury } \\
\hline Crude SHR & 1 (Reference) & $1.73(1.16-2.58)$ \\
\hline Adjusted SHR* & 1 (Reference) & $1.85(1.23-2.79)$ \\
\hline \multicolumn{3}{|c|}{ Chronic kidney disease } \\
\hline Crude SHR & 1 (Reference) & $1.72(1.50-1.97)$ \\
\hline Adjusted SHR* & 1 (Reference) & $1.73(1.51-1.98)$ \\
\hline \multicolumn{3}{|c|}{ End-stage renal disease } \\
\hline Crude SHR & 1 (Reference) & $2.92(2.13-4.00)$ \\
\hline Adjusted SHR* & 1 (Reference) & $2.91(2.11-4.00)$ \\
\hline \multicolumn{3}{|c|}{$\begin{array}{l}\text { Note: } \mathrm{CHF}=\text { congestive heart failure, } \mathrm{Cl}=\text { confidence interval, } \mathrm{COPD}=\text { chronic obstructive pulmonary disease, SHR = subhazard ratio. } \\
\text { *Covariables found significantly associated with acute kidney injury, chronic kidney disease and end-stage renal disease in the univariable } \\
\text { competing-risks regression model were further examined by the multivariable competing-risks regression model. } \\
\text { - Adjusted SHR for acute kidney injury by propensity score matching was calculated by Cox proportional hazard regression and adjusted for } \\
\text { age, sex and comorbidities of diabetes, hypertension, CHF and admission to intensive care unit. } \\
\text { Adjusted SHR for chronic kidney disease by propensity score matching was calculated by Cox proportional hazard regression and adjusted } \\
\text { for age, sex and comorbidities of diabetes, hypertension, hyperlipidemia, CHF and COPD. } \\
\text { Adjusted SHR for end-stage renal disease by propensity score matching was calculated by Cox proportional hazard regression and adjusted } \\
\text { for age, sex, Charlson Comorbidity Index score and comorbidities of diabetes, hypertension, hyperlipidemia, CHF and COPD. }\end{array}$} \\
\hline
\end{tabular}

Table 4: Predictors of de novo acute kidney injury, chronic kidney disease and end-stage renal disease by Cox regression model among propensity score-matched patients with acute critical illness compared with those without

\begin{tabular}{|c|c|c|c|c|c|c|}
\hline \multirow[b]{2}{*}{ Variable } & \multicolumn{2}{|c|}{ Acute kidney injury } & \multicolumn{2}{|c|}{ Chronic kidney disease } & \multicolumn{2}{|c|}{ End-stage renal disease } \\
\hline & $\begin{array}{l}\text { Crude HR } \\
(95 \% \mathrm{CI})\end{array}$ & $\begin{array}{c}\text { Adjusted HR } \\
\text { (95\% CI) }\end{array}$ & $\begin{array}{l}\text { Crude HR } \\
(95 \% \mathrm{CI})\end{array}$ & $\begin{array}{c}\text { Adjusted HR } \\
\text { (95\% CI) }\end{array}$ & $\begin{array}{c}\text { Crude HR } \\
(95 \% \mathrm{CI})\end{array}$ & $\begin{array}{c}\text { Adjusted HR } \\
(95 \% \mathrm{CI})\end{array}$ \\
\hline Critical illness & $2.65(1.62-4.32)$ & $2.92(1.79-4.78)$ & $1.72(1.50-1.98)$ & $1.81(1.57-2.09)$ & $3.49(2.43-5.01)$ & $3.61(2.50-5.20)$ \\
\hline Age (per 1-year increase) & $1.03(1.01-1.04)$ & $1.02(1.00-1.04)$ & $1.03(1.03-1.04)$ & $1.02(1.02-1.03)$ & $1.00(0.99-1.01)$ & $0.99(0.98-1.01)$ \\
\hline Sex (women v. men) & $0.76(0.49-1.20)$ & $0.86(0.54-1.37)$ & $1.17(1.02-1.35)$ & $1.36(1.17-1.57)$ & $0.65(0.48-0.90)$ & $0.87(0.63-1.21)$ \\
\hline \multicolumn{7}{|l|}{$\mathrm{CCl}$ score } \\
\hline 0 & 1 (Reference) & 1 (Reference) & 1 (Reference) & 1 (Reference) & 1 (Reference) & 1 (Reference) \\
\hline 1 & $1.04(0.64-1.69)$ & $0.93(0.55-1.58)$ & $1.22(1.05-1.41)$ & $0.91(0.77-1.07)$ & $0.78(0.56-1.09)$ & $0.92(0.64-1.31)$ \\
\hline 2 or more & $1.41(0.71-2.79)$ & $1.04(0.50-2.19)$ & $1.38(1.11-1.71)$ & $0.86(0.68-1.09)$ & $0.59(0.32-1.11)$ & $0.66(0.34-1.28)$ \\
\hline \multicolumn{7}{|l|}{ Comorbidity } \\
\hline Diabetes & $2.89(1.80-4.64)$ & $2.68(1.62-4.44)$ & $3.00(2.59-3.47)$ & $2.50(2.14-2.92)$ & $10.1(7.26-14.0)$ & $8.32(5.83-11.9)$ \\
\hline Cirrhosis & $1.06(0.63-1.77)$ & $1.01(0.59-1.72)$ & $1.14(0.97-1.33)$ & $0.99(0.84-1.16)$ & $1.17(0.82-1.67)$ & $0.94(0.66-1.36)$ \\
\hline Hypertension & $2.48(1.49-4.13)$ & $1.67(0.93-3.00)$ & $3.07(2.60-3.62)$ & $1.93(1.60-2.32)$ & $2.81(1.95-4.06)$ & $1.98(1.29-3.04)$ \\
\hline Hyperlipidemia & $1.12(0.68-1.84)$ & $0.73(0.43-1.25)$ & $1.70(1.47-1.96)$ & $1.11(0.95-1.29)$ & $2.40(1.75-3.29)$ & $1.14(0.81-1.60)$ \\
\hline Coronary artery disease & $1.33(0.83-2.12)$ & $0.79(0.46-1.33)$ & $1.62(1.41-1.87)$ & $0.94(0.80-1.09)$ & $1.14(0.81-1.60)$ & $0.80(0.55-1.16)$ \\
\hline $\mathrm{CHF}$ & $3.36(1.96-5.77)$ & $2.91(1.59-5.33)$ & $2.25(1.86-2.72)$ & $1.58(1.29-1.93)$ & $1.98(1.25-3.14)$ & $2.23(1.35-3.66)$ \\
\hline COPD & $1.09(0.64-1.85)$ & $0.75(0.42-1.34)$ & $1.55(1.34-1.80)$ & $1.10(0.93-1.29)$ & $0.55(0.34-0.88)$ & $0.58(0.35-0.95)$ \\
\hline Cancer & $2.32(1.11-4.84)$ & $2.06(0.97-4.37)$ & $1.07(0.79-1.47)$ & $1.02(0.75-1.40)$ & $0.73(0.30-1.78)$ & $0.84(0.34-2.06)$ \\
\hline Admission to ICU & $1.15(0.63-2.09)$ & $0.47(0.19-1.13)$ & $1.01(0.83-1.22)$ & $0.91(0.72-1.15)$ & $0.74(0.45-1.22)$ & $0.54(0.28-1.01)$ \\
\hline Mechanical ventilation & $2.66(1.44-4.94) \ddagger$ & $4.67(1.86-11.7) \ddagger$ & $1.12(0.86-1.46)$ & $1.20(0.87-1.66)$ & $1.09(0.57-2.07)$ & $2.00(0.89-4.49)$ \\
\hline
\end{tabular}

Note: $\mathrm{CCl}=$ Charlson Comorbidity Index, $\mathrm{CHF}=$ congestive heart failure, $\mathrm{Cl}=$ confidence interval, $\mathrm{COPD}=$ chronic obstructive pulmonary disease, $\mathrm{HR}=$ hazard ratio, $\mathrm{ICU}=$ intensive care unit . 
critical illness cohort than in the control cohort (9.45 v. 2.35 per 10000 person-years, incidence rate ratio [IRR] 4.02, adjusted hazard ratio [aHR] 2.84, 95\% Cl 1.95-4.15). Notably, 1225 patients in the acute critical illness cohort developed chronic kidney disease, with an incidence rate of 78.3 per 10000 person-years, also significantly higher than controls (IRR 2.08, aHR 1.81, 95\% $\mathrm{Cl}$ 1.61-2.04). Furthermore, 335 patients in the acute critical illness cohort developed end-stage renal disease, with an incidence rate of 21.0 per 10000 person-years, significantly higher than controls (IRR 4.27, aHR 3.26, 95\% CI 2.52-4.22). Results were similar in the propensity-matched subset for acute kidney injury (aHR 2.92, 95\% Cl 1.78-4.77), chronic kidney disease (aHR $1.81,95 \% \mathrm{Cl} 1.57-2.08$ ) and end-stage renal disease (aHR 3.60, $95 \% \mathrm{Cl} 2.50-5.18)$.

Figure 2 shows the cumulative risks of renal outcomes for the propensity score-matched cohorts followed for more than
12 years. The risks were significantly higher in the acute critical illness cohort than in the control cohort (log-rank test, $p<0.001$ ). Notably, the incidence of chronic kidney disease prevailed over that of end-stage renal disease and acute kidney injury in the acute critical illness cohort. In the competing risk regression model, the risks of acute kidney injury, chronic kidney disease and end-stage renal disease remained higher in the propensity score-matched acute critical illness cohort (adjusted subhazard ratios [aSHRs] 1.85, 95\% Cl 1.23-2.79; aSHRs 1.73, 95\% Cl 1.511.98; and aSHRs $2.91,95 \% \mathrm{Cl} 2.11-4.00$, respectively; Table 3) than in the controls.

Table 4 shows the predictors of acute kidney injury, chronic kidney disease and end-stage renal disease in the propensity score-matched cohorts. Among these comorbidities, diabetes, hypertension and congestive heart failure were universal risk factors for acute kidney injury, chronic kidney disease and end-stage

Table 5: Comparisons of incidence and hazard ratios of acute kidney injury, chronic kidney disease and end-stage renal disease in patients with different subtypes of critical illness compared with those without critical illness in the propensity score-matched cohorts

\begin{tabular}{|c|c|c|c|c|}
\hline Variable & No. of events & Person-years & Rate* $^{\star}$ & Adjusted HR $\dagger(95 \% \mathrm{Cl})$ \\
\hline
\end{tabular}

\section{Acute kidney injury}

Critical illness

$\begin{array}{lccccc}\text { None } & 23 & 76747 & 3.00 & 1 \text { (Reference) } & 1 \text { (Reference) } \\ \text { Septicemia, septic shock } & 42 & 34889 & 12.0 & 4.09(2.46-6.80) & 4.93(2.91-8.35) \\ \text { Hemorrhagic stroke } & 0 & 2368 & 0.00 & - & - \\ \text { Ischemic stroke } & 10 & 24472 & 4.09 & 1.37(0.65-2.89) & 1.22(0.57-2.63) \\ \text { AMI } & 1 & 2623 & 3.81 & 1.29(0.18-9.58) & 1.73(0.23-13.2)\end{array}$

\section{Chronic kidney disease}

Critical illness

$\begin{array}{lccccc}\text { None } & 325 & 75818 & 42.9 & 1 \text { (Reference) } & 1 \text { (Reference) } \\ \text { Septicemia, septic shock } & 305 & 34092 & 89.5 & 2.12(1.81-2.48) & 2.46(2.10-2.90) \\ \text { Hemorrhagic stroke } & 15 & 2309 & 65.0 & 1.53(1.91-20.57) & 1.63(0.97-2.76) \\ \text { Ischemic stroke } & 147 & 24074 & 61.1 & 1.43(1.18-1.74) & 1.22(0.99-1.50) \\ \text { AMI } & 14 & 2593 & 54.0 & 1.27(0.74-2.17) & 1.31(0.76-2.27) \\ \text { Hemorrhagic shock } & 3 & 3278 & 9.15 & 0.21(0.07-0.67) & 0.41(0.13-1.29) \\ \text { End-stage renal disease } & & & & 1 \text { (Reference) } \\ \text { Critical illness } & 39 & & & 5.29(3.63-7.71) & 1 \text { (Reference) } \\ \text { None } & 89 & 76717 & 5.08 & 5.38(3.65-7.94) \\ \text { Septicemia, septic shock } & 2 & 34848 & 25.5 & 1.68(0.41-6.97) & 1.90(0.45-8.03) \\ \text { Hemorrhagic stroke } & 23 & 2365 & 8.46 & 1.88(1.13-3.15) & 1.64(0.95-2.84) \\ \text { Ischemic stroke } & 2 & 24447 & 9.41 & 1.53(0.37-6.32) & 1.88(0.44-8.01) \\ \text { AMI } & & 2627 & 7.61 & & \end{array}$

Note: $\mathrm{AMI}=$ acute myocardial infarction, $\mathrm{CHF}=$ congestive heart failure, $\mathrm{Cl}=$ confidence interval, $\mathrm{COPD}=$ chronic obstructive pulmonary disease, $\mathrm{HR}=$ hazard ratio . *Incidence rate, per 10000 person-years.

†Covariables found significantly associated with acute kidney injury, chronic kidney disease, and end-stage renal disease in the univariable Cox proportional regression model were further examined by the multivariable Cox proportional regression model:

- Adjusted HR for acute kidney injury by propensity score matching was calculated by Cox proportional hazard regression and adjusted for age and comorbidities of diabetes, CHF, cancer, hypertension, admission to intensive care unit and mechanical ventilation.

- Adjusted HR for chronic kidney disease by propensity score matching was calculated by Cox proportional hazard regression and adjusted for age, sex, Charlson Comorbidity Index score and comorbidities of diabetes, hypertension, hyperlipidemia, coronary artery disease, CHF, COPD, cancer and mechanical ventilation.

- Adjusted HR for end-stage renal disease by propensity score matching was calculated by Cox proportional hazard regression and adjusted for sex and comorbidities of diabetes, hypertension, hyperlipidemia, CHF, COPD and mechanical ventilation. 
renal disease in our cohorts. Mechanical ventilation was associated with acute kidney injury (aHR 2.56, 95\% Cl 1.37-4.79), but not with chronic kidney disease and end-stage renal disease. Next, patients were stratified by the primary causes of critical illnesses, and renal outcome was compared for the propensity score-matched cohorts (Table 5). Among them, septicemia and septic shock were the strongest risk factor for acute kidney injury (aHR 4.93, 95\% Cl 2.91-8.35), chronic kidney disease (aHR 2.46, $95 \% \mathrm{Cl} 2.10-2.90$ ) and end-stage renal disease (aHR 5.38, 95\% Cl 3.65-7.94).

\section{Secondary outcome}

We analyzed mortality after the development of acute kidney injury, chronic kidney disease and end-stage renal disease in patients with acute critical illness and nonacute critical illness in both the frequency-matched and propensity score-matched cohorts (Table 6). Patients with acute critical illness had a higher risk of death after developing chronic kidney disease (aHR 2.16, $95 \% \mathrm{Cl} 1.67-2.80)$ and after progression to end-stage renal disease (aHR 3.37, 95\% Cl 2.07-5.49) than did the controls in the frequency-matched cohorts, with similar results seen in the propensity-matched cohorts. Figure 3 shows the higher cumulative risk of death after the development of chronic kidney disease and end-stage renal disease in the frequency-matched acute critical illness cohorts than that of the controls. However, we observed no significant difference in the risk of mortality after patients in the frequency-matched cohort had developed acute kidney injury.

\section{Interpretation}

We investigated long-term risks of renal sequelae and subsequent mortality in patients with acute critical illness without pre-existing renal disease. The acute critical illness cohort had

Table 6: Incidence and hazard ratios of mortality after patients developed acute kidney injury, chronic kidney disease or end-stage renal disease in the critical illness cohorts compared with those in the controls, in age- and sex-matched and propensity score-matched cohorts

\section{Critical illness}

\section{Age- and sex-matched}

\section{Variable}

\section{After acute kidney injury}

No. of deaths

Rate*

Crude HR $(95 \% \mathrm{Cl})$

Adjusted HR† $(95 \% \mathrm{Cl})$

\section{After chronic kidney disease}

Death

Rate* $^{*}$

No

23

1 (Reference)

1 (Reference)

57

15.6

$1.19(0.73-1.94)$

$1.14(0.60-2.18)$

293

8.04

3.68

1 (Reference)

$2.19(1.80-2.65)$

1 (Reference)

$2.16(1.67-2.80)$

Crude HR $(95 \% \mathrm{Cl})$

Adjusted HR† $(95 \% \mathrm{Cl})$
Propensity score-matched

No

Yes

5

17

13.3

1 (Reference)

$1.04(0.37-2.88)$

1 (Reference)

$1.52(0.45-5.19)$
After end-stage renal disease

Death

Rate $^{*}$

Crude HR $(95 \% \mathrm{Cl})$

Adjusted HR† $(95 \% \mathrm{Cl})$
36

9.1

1 (Reference)

1 (Reference)
150

25.2

$2.56(1.78-3.68)$

3.37 (2.07-5.49)
35

3.62

1 (Reference)

1 (Reference)

5.89

1 (Reference)

$4.76(1.73-13.1)$

1 (Reference)
121

8.49

$2.37(1.63-3.46)$

$2.41(1.63-3.56)$
$.73(1.88-17.5)$

Note: $\mathrm{CAD}=$ coronary artery disease, $\mathrm{CCl}=$ Charlson Comorbidity Index, $\mathrm{CHF}=$ congestive heart failure, $\mathrm{Cl}=$ confidence interval, $\mathrm{COPD}=$ chronic obstructive pulmonary disease, $\mathrm{HR}=$ hazard ratio.

*Incidence rate, per 10000 person-years.

tCovariables found significantly associated with mortality in the univariable Cox proportional regression model were further examined by the multivariable Cox proportional regression model:

- Adjusted HR for acute kidney injury by age- and sex-matching was calculated by Cox proportional hazard regression and adjusted for age, sex, CCI score and comorbidities of diabetes, cirrhosis, hypertension, hyperlipidemia, cancer, CAD, CHF, COPD, admission to intensive care unit and mechanical ventilation. Adjusted HR for acute kidney injury by propensity score matching was calculated by Cox proportional hazard regression and adjusted for age, and comorbidities of diabetes, CHF, cancer, hypertension, admission to intensive care unit and mechanical ventilation.

- Adjusted HR for chronic kidney disease by age- and sex-matching was calculated by Cox proportional hazard regression and adjusted for age, sex, CCl score and comorbidities of diabetes, cirrhosis, hypertension, hyperlipidemia, cancer, CAD, CHF, COPD, admission to intensive care unit and mechanical ventilation. Adjusted HR for chronic kidney disease by propensity score matching was calculated by Cox proportional hazard regression and adjusted for age, sex, $\mathrm{CCl}$ score and comorbidities of diabetes, hypertension, hyperlipidemia, CAD, CHF, COPD, cancer and mechanical ventilation.

- Adjusted HR for end-stage renal disease by age- and sex-matching was calculated by Cox proportional hazard regression and adjusted for comorbidities of diabetes, hypertension, hyperlipidemia, CAD, CHF, COPD, admission to intensive care unit and mechanical ventilation. Adjusted HR for end-stage renal disease by propensity score matching was calculated by Cox proportional hazard regression and adjusted for sex and comorbidities of diabetes, hypertension, hyperlipidemia, CHF, COPD and mechanical ventilation. 
A

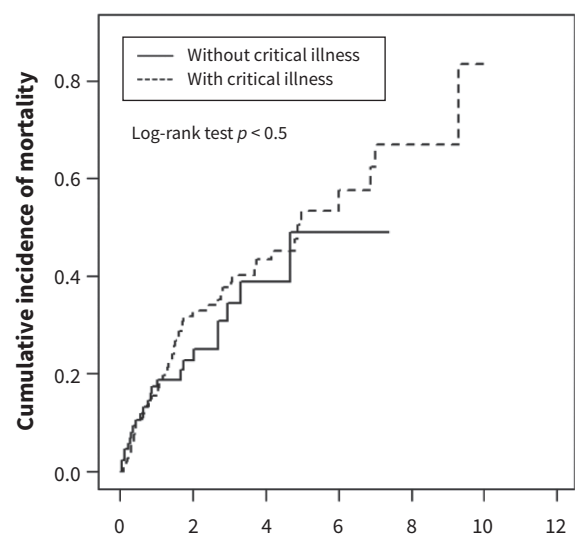

\begin{tabular}{rccccccc} 
& \multicolumn{7}{c}{ Years } \\
Critical illness, no. at risk & 151 & 62 & 35 & 11 & 4 & 0 & 0 \\
Mortality events & 0 & 41 & 8 & 4 & 3 & 1 & 1 \\
Without critical illness, no. at risk & 83 & 25 & 6 & 3 & 1 & 0 & 0 \\
Mortality events & 0 & 17 & 4 & 1 & 0 & 0 & 0
\end{tabular}

\section{B}

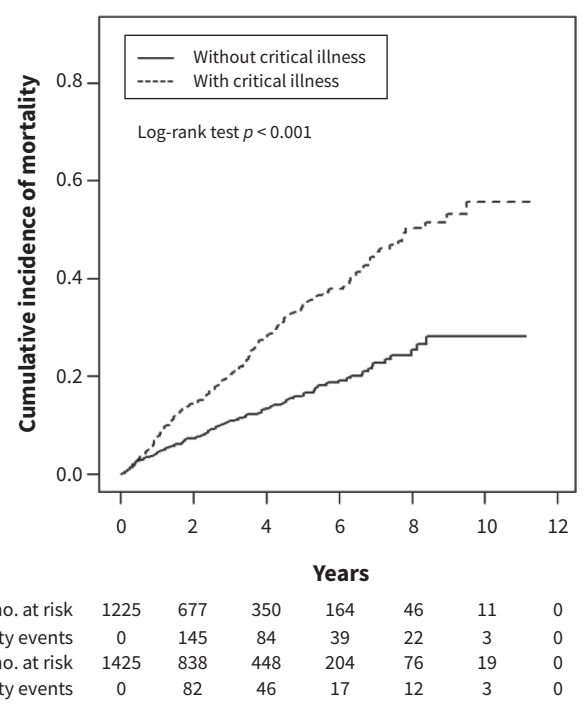

C

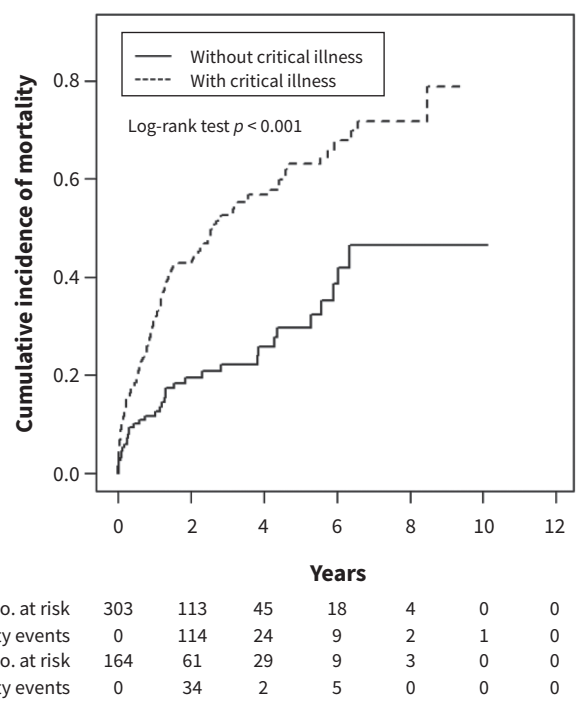

Figure 3: Cumulative incidence of death after development of acute kidney disease (A), chronic kidney disease (B) and end-stage renal disease (C) in the age- and sex-matched cohorts. an increased risk of renal complications, with the highest incidence of chronic kidney disease, followed by end-stage renal disease and acute kidney injury. Among the primary causes of critical illnesses, septicemia and septic shock were the strongest risk factors for acute kidney injury, chronic kidney disease and end-stage renal disease. These results showed that patients with acute critical illness without apparent underlying renal disease - a group traditionally considered to be at low risk for renal disease - have clinically relevant long-term renal risks. In addition, the status of acute critical illness has a negative impact on mortality, modified by subsequent development of renal complications.

Recent studies have shown that milder forms of acute kidney injury and even recovery of renal function after acute kidney injury are associated with both short- and long-term mortality. ${ }^{16,29,30}$ Bucaloiu and colleagues advised that even a reversible acute kidney injury event is a risk factor for death and de novo chronic kidney disease (hazard ratio [HR] 1.91, compared with the nonacute kidney injury group). ${ }^{30}$ In our study, patients with acute critical illness might have renal recovery upon discharge, and showed a similar risk of chronic kidney disease (aHR: 1.81). The Kidney Disease Improving Global Outcomes guideline on acute kidney injury recommends assessing patients 2 months after acute kidney injury to evaluate the completeness of disease resolution and to detect either new-onset or worsening chronic kidney disease. ${ }^{2}$ Thus, we propose surveillance of renal function at 30-90 days and then at least yearly in patients with acute critical illness even when they do not have pre-existing renal disease. ${ }^{18}$

Among traditional risk factors for chronic kidney disease and end-stage renal disease, hypertension, diabetes and congestive heart failure were prevalent in our acute critical illness cohorts. Our results showed that acute physiologic abnormalities and mechanical ventilation are predictors of de novo acute kidney injury. To date, the Acute Physiologic and Chronic Health Evaluation (APACHE) II scoring system has been widely used for predicting survival and recovery of renal function in studies of acute kidney injury. ${ }^{31}$ Although our study did not evaluate the APACHE II score owing to database limitation, we used mechanical ventilation as a proxy for illness severity as it is an independent factor for mortality in critical illness patients. ${ }^{8}$ The US Renal Data System (2006) indicates the risk of acute kidney injury in patients who are older, have received ICU care and have multiple organ dysfunction. ${ }^{32}$ This evidence shows that acute kidney injury is a complex syndrome reflecting underlying disease burden; subsequent chronic kidney disease and endstage renal disease may account for increased mortality in patients with acute critical illness.

Sepsis is the leading cause of acute kidney injury, accounting for $45 \%-70 \%$ of all acute kidney injury events in patients in the ICU. ${ }^{8,33}$ Sepsis was associated with higher aberrations in hemodynamics, illness severity and need for mechanical ventilation. ${ }^{34}$ Circulatory shock and infection are important risk factors for acute kidney injury in patients in the ICU. ${ }^{35}$ Bellomo and colleagues proposed that even a single episode of septic acute kidney injury is associated with increased risk of developing 
chronic kidney disease. ${ }^{36}$ In our study, patients with sepsis had higher risks of renal complications than did those with cardiovascular diseases. We suspect that although patients may experience renal recovery from septic acute kidney injury, they may still be predisposed to subsequent chronic kidney disease and end-stage renal disease. Epidemiological studies have reported an association between pneumonia and subsequent acute kidney injury and end-stage renal disease, supporting our findings that infections may trigger not only acute but also chronic renal damage. ${ }^{37,38}$

\section{Limitations}

The study has potential limitations. First, retrospective cohort studies have various biases because of the possible unmeasured factors related to both exposure and outcomes. To limit the potential for confounding factors, we used propensity-score matching across a wide range of clinical characteristics to establish the cohorts. Second, the claim database was limited because biological information such as laboratory data (e.g., glomerular filtration rate as the overall index of renal function) were unavailable. Because physicians were reimbursed for chronic kidney disease and end-stage renal disease care after quarterly reporting of longitudinal estimated glomerular filtration rate and urinary screening data, this strengthens the validity of the renal disease diagnosis in the database we used. Finally, some patients with pre-existing unrecognized renal dysfunction could have been included in the study and control cohorts. Nevertheless, based on the consistent results obtained using various approaches, our study provides important evidence to support the increased risk of renal complications in patients with acute critical illness.

\section{Conclusion}

Our study showed higher risks of acute kidney injury, chronic kidney disease and end-stage renal disease in patients with acute critical illness without pre-existing renal disease. Developing chronic kidney disease and end-stage renal disease further increased subsequent mortality risks. Nephrological surveillance of patients with acute critical illness, particularly those with sepsis and ischemic stroke, should be an essential part of their follow-up care.

\section{References}

1. KDIGO clinical practice guideline for acute kidney injury. Kidney Int Suppl 2012;2:1-138.

2. Poukkanen M, Vaara ST, Pettila V, et al.; FINNAKI Study Group. Acute kidney injury in patients with severe sepsis in Finnish intensive care units. Acta Anaesthesiol Scand 2013;57:863-72.

3. Zambetti BR, Thomas F, Hwang I, et al. A web-based tool to predict acute kidney injury in patients with ST-elevation myocardial infarction: Development, internal validation and comparison. PLoS One 2017;12:e0181658.

4. Wang $D$, Guo $Y$, Zhang $Y$, et al. Epidemiology of acute kidney injury in patients with stroke: a retrospective analysis from the neurology ICU. Intern Emerg Med 2018:13:17-25.

5. Hsu RK, McCulloch CE, Heung M, et al. Exploring potential reasons for the temporal trend in dialysis-requiring AKI in the United States. Clin J Am Soc Nephrol 2016;11:14-20.
6. Hsu RK, McCulloch CE, Dudley RA, et al. Temporal changes in incidence of dialysisrequiring AKI. J Am Soc Nephrol 2013;24:37-42.

7. Macedo E, Mehta RL. Epidemiology, diagnosis, and therapy of acute kidney injury. In: Thomas MC, Robert WS, editors. Schrier's diseases of the kidney, ninth edition. Philadelphia (PA): Lippincott Williams \& Wilkins; 2012:795.

8. Uchino S, Kellum JA, Bellomo R, et al. Acute renal failure in critically ill patients: a multinational, multicenter study. JAMA 2005;294:813-8.

9. Lo LJ, Go AS, Chertow GM, et al. Dialysis-requiring acute renal failure increases the risk of progressive chronic kidney disease. Kidney Int 2009;76:893-9.

10. Coca SG, Singanamala S, Parikh CR, et al. Chronic kidney disease after acute kidney injury: a systematic review and meta-analysis. Kidney Int 2012;81:442-8.

11. Wald R, Quinn RR, Luo J, et al. Chronic dialysis and death among survivors of acute kidney injury requiring dialysis. JAMA 2009;302:1179-85.

12. Gammelager H, Christiansen CF, Johansen MB, et al. Five-year risk of end-stage renal disease among intensive care patients surviving dialysis-requiring acute kidney injury: a nationwide cohort study. Crit Care 2013;17:R145.

13. Levey AS, Stevens LA, Schmid CH, et al. A new equation to estimate glomerular filtration rate. Ann Intern Med 2009;150:604-12.

14. Levey AS, Schoolwerth AC, Burrows NR, et al. Comprehensive public health strategies for preventing the development, progression, and complications of CKD: report of an expert panel convened by the Centers for Disease Control and Prevention. Am J Kidney Dis 2009;53:522-35

15. Ishani A, Xue JL, Himmelfarb J, et al. Acute kidney injury increases risk of ESRD among elderly. J Am Soc Nephrol 2009;20:223-8.

16. Rimes-Stigare $\mathrm{C}$, Frumento $\mathrm{P}$, Bottai $\mathrm{M}$, et al. Long-term mortality and risk factors for development of end-stage renal disease in critically ill patients with and without chronic kidney disease. Crit Care 2015;19:383.

17. Chawla LS, Bellomo R, Bihorac A, et al. Acute kidney disease and renal recovery: consensus report of the Acute Disease Quality Initiative (ADQI) 16 Workgroup. Nat Rev Nephrol 2017;13:241-57.

18. Goldstein SL, Chawla L, Ronco C, et al. Renal recovery. Crit Care 2014;18:301.

19. National Health Insurance Research Database, Taiwan. Available: http://nhird. nhri.org.tw/en/index.html (accessed 2018 Aug. 3).

20. National Health Insurance Administration. National Health Insurance annual report 2014-2015. Taiwan: Ministry of Health and Welfare; 2014.

21. Longitudinal Health Insurance Database (LHID), data subsets, Database NHIR Taiwan. Available: https://nhird.nhri.org.tw/en/Data_Subsets.html (accessed 2018 Aug. 3).

22. Inker LA, Astor BC, Fox CH, et al. KDOQI US commentary on the 2012 KDIGO clinical practice guideline for the evaluation and management of CKD. Am J Kidney Dis 2014;63:713-35.

23. Frenkel WJ, Jongerius EJ, Mandjes-van Uitert MJ, et al. Validation of the Charlson Comorbidity Index in acutely hospitalized elderly adults: a prospective cohort study. J Am Geriatr Soc 2014;62:342-6.

24. National Kidney Foundation. K/DOQI clinical practice guidelines for chronic kidney disease: evaluation, classification, and stratification. Am J Kidney Dis 2002;39:(2 Suppl 1)1-266.

25. Wu VC, Wang CY, Shiao CC, et al.; National Taiwan University Study Group on Acute Renal Failure; Taiwan Anti-Mycobacteria Investigation Group. Increased risk of active tuberculosis following acute kidney injury: a nationwide, populationbased study. PLoS One 2013;8:e69556.

26. Cheng CL, Kao YH, Lin SJ, et al. Validation of the National Health Insurance Research Database with ischemic stroke cases in Taiwan. Pharmacoepidemiol Drug Saf 2011;20:236-42.

27. Parsons LS; Ovation Research Group, Seattle, Washington. Performing a 1:N Case-control match on propensity score [poster]. Proceedings from SUGI 29; 2004 May 9-12; Montréal. Available: www2.sas.com/proceedings/sugi29/165-29. pdf (accessed 2018 Aug. 3).

28. Fine JP, Gray RJ. A proportional hazards model for the subdistribution of a competing risk. J Am Stat Assoc 1999;94:496-509. 
29. Pannu N, James M, Hemmelgarn B, et al. Association between AKI, recovery of renal function, and long-term outcomes after hospital discharge. Clin J Am Soc Nephrol 2013;8:194-202.

30. Bucaloiu ID, Kirchner HL, Norfolk ER, et al. Increased risk of death and de novo chronic kidney disease following reversible acute kidney injury. Kidney Int 2012;81:477-85.

31. Parker RA, Himmelfarb J, Tolkoff-Rubin N, et al. Prognosis of patients with acute renal failure requiring dialysis: results of a multicenter study. Am J Kidney Dis 1998;32:432-43

32. Xue JL, Daniels F, Star RA, et al. Incidence and mortality of acute renal failure in Medicare beneficiaries, 1992 to 2001. J Am Soc Nephrol 2006;17:1135-42.

33. Bagshaw SM, Laupland KB, Doig CJ, et al. Prognosis for long-term survival and renal recovery in critically ill patients with severe acute renal failure: a population-based study. Crit Care 2005;9:R700-9.
34. Bagshaw SM, Uchino S, Bellomo R, et al. Septic acute kidney injury in critically ill patients: clinical characteristics and outcomes. Clin J Am Soc Nephrol 2007;2:431-9.

35. de Mendonça A, Vincent JL, Suter PM, et al. Acute renal failure in the ICU: risk factors and outcome evaluated by the SOFA score. Intensive Care Med 2000;26:915-21.

36. Bellomo R, Kellum JA, Ronco C, et al. Acute kidney injury in sepsis. Intensive Care Med 2017;43:816-28.

37. Lin TY, Chen YG, Lin CL, et al. Increased risk of acute kidney injury following pneumococcal pneumonia: a nationwide cohort study. PLoS One 2016;11:e0158501.

38. Huang ST, Lin CL, Chang YJ, et al. Pneumococcal pneumonia infection is associated with end-stage renal disease in adult hospitalized patients. Kidney Int 2014;86:1023-30.

\section{Competing interests: None declared.}

This article has been peer reviewed

Affiliations: Division of Nephrology, Taichung Veterans General Hospital, Taichung, Taiwan (Huang, Chuang); Graduate Institute of Public Health, China Medical University, Taichung, Taiwan (Huang, Chuang); Division of Nephrology, Ministry of Health and Welfare Chiayi Hospital, Chiayi, Taiwan (Ke); Management Office for Health Data, China Medical University Hospital, Taichung, Taiwan (Lin); College of Medicine, China Medical University, Taichung, Taiwan (Lin); Graduate Institute of Biomedical Sciences and School of Medicine, College of Medicine, China Medical University, Taichung, Taiwan (Kao); Department of Nuclear Medicine and PET Center, China Medical University, Taichung, Taiwan (Kao); Department of Bioinformatics and Medical Engineering, Asia University, Taichung, Taiwan (Kao).
Contributors: Shih-Ting Huang and Chia-Hung Kao were responsible for the conception and design of the work. Chia-Hung Kao was responsible for providing the study materials. All authors contributed to the acquisition analysis and interpretation of the data. All of the authors drafted the manuscript, revised it critically for important intellectual content, gave approval of the final version to be published and agreed to be accountable for all aspects of the work.

Funding: This work was supported by grants from the Ministry of Health and Welfare, Taiwan (MOHW107-TDU-B-212-123004), China Medical University Hospital, Academia Sinica Stroke Biosignature Project (BM10701010021), MOST Clinical Trial Consortium for Stroke (MOST 106-2321-B-039-005-)." Tseng-Lien Lin Foundation, Taichung, Taiwan, and Katsuzo and Kiyo Aoshima Memorial Funds, Japan. The funders had no role in the study design, data collection and analysis, publication decision, or manuscript drafting. No additional external funding was received for this study.

Data sharing: The data set used in this study is held by the Taiwan Ministry of Health and Welfare (MOHW). The Ministry of Health and Welfare must approve applications to access these data. Any researcher interested in accessing this data set can submit an application form to MOHW to request access. Please contact the staff of MOHW (stcarolwu@mohw. gov.tw) for further assistance. Taiwan Ministry of Health and Welfare Address: No.488, Sec. 6 , Zhongxiao E. Rd., Nangang Dist., Taipei City 115, Taiwan (R.O.C.). Phone: +886-2-85906848. All relevant data are within the paper.

Accepted: July 4, 2018

Correspondence to: Chia-Hung Kao, d10040@mail.cmuh.org.tw 